\title{
A Four-Dimensional Variational System for Skillful Operational Prediction of Convective Storms
}

\author{
Nathan SNOOK ${ }^{* 1}$ and Qinghong ZHANG ${ }^{2}$ \\ ${ }^{1}$ Center for the Analysis and Prediction of Storms, University of Oklahoma, Norman, OK 73072, USA \\ ${ }^{2}$ Department of Atmospheric \& Oceanic Sciences, School of Physics, Peking University, Beijing 100871, China
}

Citation: Snook, N., and Q. H. Zhang, 2016: A four-dimensional variational system for skillful operational prediction of convective storms. Adv. Atmos. Sci., 33(10), 1102-1103, doi: 10.1007/s00376-016-6170-3.

Forecasting convective storms using NWP models is an important goal and a highly active area of ongoing research. Skillful and reliable NWP of convective storms could allow for severe weather warnings with longer lead times, as operational forecasters begin to incorporate convective-scale forecasts into severe weather forecast operations (Stensrud et al., 2009, 2013). This would then provide vulnerable individuals and industries with more time to seek shelter and/or mitigate the impact of severe weather hazards.

The importance of data assimilation (DA) of radar observations for convective-scale NWP is widely acknowledged; consequently, a wide variety of DA methods have been explored and studied. Both variational and ensemble DA methods (as well as hybrid methods), have been successfully applied in convective-scale NWP. Understanding the relative merits and challenges associated with different DA methods, and the relative impacts of different datasets, is vital for advancing the science underpinning the prediction of convective storms.

Some of these issues are addressed by Chen et al. (2016) in their new study (Page 1106-1119, this issue), as they investigate the impacts of assimilating both surface and radar observations using a four-dimensional variational (4DVAR) DA system. While 4DVAR requires an adjoint model, which can be difficult to develop and computationally expensive to run, a 4DVAR system has certain advantages over 3DVAR and EnKF (ensemble Kalman filter) systems, including the ability to assimilate asynchronous observations including time correlations (Kalnay et al., 2007). The advantages of 4DVAR make it particularly desirable for convective-scale NWP.

In recent years, rapid advances have been made in stormscale DA and prediction. 3DVAR, 4DVAR, and EnKF DA systems have been applied successfully to a wide range of cases and observational datasets, and are a key component of efforts within the United States (and around the world) to develop a warn-on-forecast weather warning system (Stensrud et al., 2013). Assimilation of radar data has been found to be

\footnotetext{
* Corresponding author: Nathan SNOOK

Email: nsnook@ou.edu
}

vital for accurate storm prediction (e.g., Dawson et al., 2012; Snook et al., 2015; Yussouf et al., 2015), and the assimilation of surface observations has also been shown to be beneficial (e.g., Schenkman et al., 2011). Most of these studies have used experimental 3DVAR, EnKF, or hybrid ensemble/variational DA systems, and have largely been focused on convection occurring over the southern great plains of the United States or in Europe.

In contrast, Chen et al. (2016) apply a 4DVAR Doppler radar analysis system (VDRAS) to assimilate radar and surface observations in a cloud forecast run with $3 \mathrm{~km}$ grid spacing. In its original implementation (Sun and Crook, 1997), VDRAS used a separate Barnes objective analysis procedure to analyze surface observations, and this analysis serves as the background for 4DVAR assimilation of radar observations. In this study, Chen et al. (2016) modify VDRAS, to assimilate surface and radar observations simultaneously using 4DVAR, removing the need for a two-step assimilation process and improving the resulting analyses. To examine the capabilities and performance of this system, they apply it to a squall-line case study from the OPACC (Observation, Prediction and Analysis of severe Convection of China) field campaign, located over the Yangtze-Huaihe river basin in eastern China. Through a set of idealized single-observation experiments and a set of data impact experiments using real data, they demonstrate the value of assimilating both surface and radar observations via the VDRAS system in terms of improving the quality of the model state in analyses and forecasts. In their real-data experiments, they assimilate radar reflectivity and radial velocity observations from seven operational radars, including both $\mathrm{S}$-band and $\mathrm{C}$-band radars, as well as surface observations. When both radar and surface observations were assimilated, analyzed wind profiles and surface temperature evolution were found to be closer to independent observations than when radar data were omitted or when surface data were assimilated separately prior to radar data analysis using VDRAS.

The study of Chen et al. (2016) shows the promise of 4DVAR for short-term, storm-scale forecasting. The ability to efficiently assimilate large amounts of data from a variety of observational platforms (including both radar and 
surface observations) using 4DVAR, as is demonstrated by the VDRAS system used in Chen et al. (2016), is vital for successful operational storm-scale NWP. Future investigations of such 4DVAR systems, and other DA systems capable of rapidly and efficiently assimilating large volumes of weather observations, will be vital in the development of next-generation operational forecasting infrastructure.

\section{REFERENCES}

Chen, X. C., K. Zhao, J. Z. Sun, B. W. Zhou, and W. C. Lee, 2016: Assimilating surface observations in a four-dimensional variational Doppler radar data assimilation system to improve the analysis and forecast of a squall line case. Adv. Atmos. Sci., 33(10), doi: 10.1007/s00376-016-5290-0.

Dawson, D. T., II, L. J. Wicker, E. R. Mansell, and R. L. Tanamachi, 2012: Impact of the environmental low-level wind profile on ensemble forecasts of the 4 May 2007 Greensburg, Kansas, tornadic storm and associated mesocyclones. Mon. Wea. Rev., 140, 696-716, doi: 10.1175/MWR-D-11-00008.1.

Kalnay, E., H. Li, T. Miyoshi, S.-C. Yang, and J. Ballabrera-Poy, 2007: 4-D-Var or ensemble Kalman filter. Tellus A, 59, 758773.

Schenkman, A. D., M. Xue, A. Shapiro, K. Brewster, and J. D.
Gao, 2011: The analysis and prediction of the 8-9 May 2007 Oklahoma tornadic mesoscale convective system by assimilating WSR-88D and CASA radar data using 3DVAR. Mon. Wea. Rev., 139, 224-246.

Snook, N., M. Xue, and Y. Jung, 2015: Multiscale EnKF assimilation of radar and conventional observations and ensemble forecasting for a tornadic mesoscale convective system. Mon. Wea. Rev., 143, 1035-1057, doi: 10.1175/MWR-D-1300262.1.

Stensrud, D. J., and Coauthors, 2009: Convective-scale warn-onforecast system: A vision for 2020. Bull. Am. Meteor. Soc., 90, 1487-1499.

Stensrud, D. J., and Coauthors, 2013: Progress and challenges with Warn-on-Forecast. Atmos. Res., 123, 2-16, doi: 10.1016/j.atmosres.2012.04.004.

Sun, J. Z., and N. A. Crook, 1997: Dynamical and microphysical retrieval from Doppler radar observations using a cloud model and its adjoint. Part I: Model development and simulated data experiments. J. Atmos. Sci., 54, 1642-1661.

Yussouf, N., D. C. Dowell, L. J. Wicker, K. H. Knopfmeier, and D. M. Wheatley, 2015: Storm-scale data assimilation and ensemble forecasts for the 27 April 2011 severe weather outbreak in Alabama. Mon. Wea. Rev., 143, 3044-3066, doi: 10.1175/MWR-D-14-00268.1. 